\title{
Genomics for Abiotic Stress Tolerance in Crop Plants
}

\section{Shabir H Wani*}

Mountain Research Centre for Field Crops, Sher-e-Kashmir University of Agricultural Sciences and Technology of Kashmir, India

"Corresponding author: Shabir H Wani, Mountain Research Centre for Field Crops, Khudwani Anantnag-192101, Sher-e-Kashmir University of Agricultural Sciences and Technology of Kashmir, Jammu \& Kashmir, India. Tel: +919419035566; +919797001791; Email: shabirhussainwani@gmail.com

Citation: Wani HS (2017) Genomics for Abiotic Stress Tolerance in Crop Plants. Int J Genom Data Min 01: 113. DOI: $10.29011 / 2577-0616.000102$

Received Date: 24 October, 2017; Accepted Date: 25 October, 2017; Published Date: 02 November, 2017

\section{Editorial}

Abiotic stresses including extreme temperatures drought salinity water logging and mineral toxicities are responsible for crop yield loss at global level. In the present situation of universal climate change and unbalanced weather (temperature, rainfall, humidity) all over the growing season leading to the exposure of sessile plants to various imminent abiotic stresses such as drought, salinity, extreme temperatures and flooding, it is imperative to accelerate the efforts for unravelling the biochemical, physiological and molecular mechanisms underlying abiotic stress tolerance in plants. Although many initiatives have been taken up in various leading institutes worldwide but the pace of development of stress tolerant cultivars is far lagging behind the increasing pressure of abiotic stresses.

Likewise, hefty losses of grain yield in crop plants arise as a result of pathogen attack, predominantly during vulnerable stages of grain germination and development. In addition, the anticipated paucity of fresh water entails that the intensity of abiotic stresses will enhance. Consequently, there is an exigency to develop improved cultivars showing resilience toward abiotic stresses to guarantee food security and safety for the near future. Advancement accomplished via contemporary plant breeding approaches to develop abiotic stress-tolerant cultivars is slow due to multigene origins of plant adaptive responses and involvement of complex genetic mechanisms.

To endure under stress conditions, plants have evolved complex mechanisms to recognize peripheral signals that allow them to counter varying environmental conditions. These mechanisms include stress perception, signal transduction, transcriptional activation of stress-responsive target genes, and synthesis of stress-related proteins and other molecules, which support plants to muddle through adverse environmental conditions through biochemical, physiological and molecular manifestations. Significantly, understanding the relationship between plant's early stress response and downstream events to fiddle with malformed conditions is one of the splendid challenges in plant biology. Rigorous research over more than last decade has progressively unravelled the mechanisms underlying plant response toward abiotic stresses, but countless facets remain unanswered. The absolute understanding of physiological, biochemical and molecular responses and tolerance mechanisms, and detection of prospective mysterious stressresponsive pathways and genes in abiotic plant stress tolerance may contribute to better understanding of underlying molecular mechanisms.

Discoveries of novel genes and pathways, analyses of expression patterns and the determination of function of these genes during abiotic stress adaptation will provide the foundation for efficient engineering strategies with the aim to enhance abiotic stress tolerance of crop plants. The novel acquaintance gained throughout this research will facilitate in the application of stress responsive determinants and in engineering of plants with enhanced tolerance to abiotic stresses. Thus, genomics approaches such as transgenics and marker assisted breeding including high throughput DNA sequencing technologies novel nigh density mapping will certainly accelerate the present crop improvement programmes for development of abiotic stress resilient crop plants at global level. 